\title{
STUDIES ON THE SPECIFIC GRAVITY OF THE URINE ${ }^{1}$
}

\author{
BY O. H. PERRY PEPPER
}

(From the Medical Clinic of the University Hospital and the John Herr Musser Department of Research Medicine, University of Pennsylvania)

(Received for publication, May 19, 1924)

In a study of the effect of the various solids of the urine upon the specific gravity of the urine, certain relationships have been found which are interesting and perhaps significant.

The specific gravity of the urine or of any solution containing more than one solute is the summation of the several specific gravities which would result if each solid were dissolved singly to the same volume of solution. In other words, each individual solid contributes an increment which varies with the concentration of the substance. This was confirmed by experiment for urea and sodium chloride, the substances of primary importance in these studies.

At $15^{\circ} \mathrm{C}$. a 1 per cent solution of urea has a specific gravity of 1.0028; a 1 per cent solution of sodium chloride, 1.0067. In these studies the specific gravity of the urine was determined at $15^{\circ}$ with the Westphal balance, the urea estimated by the urease method, the chloride by the Volhard method. The content of urea and chloride can then be converted into terms of specific gravity and these values analyzed. Thanks are due to Mr. J. G. Camack for technical assistance

In these studies both single voidings and twenty-four hour collections were used. In the normals to be reported no effort to control diet was made and such variations in diet as occurred were spontaneous. The studies made upon normals on high or low salt or protein intake are as yet too few to report. In the nephritics studied the diets were shifted from low salt and protein to high salt and protein without altering the constancy of the relations about to be described.

For convenience we may call that part of the total specific gravity which is not accounted for by the urea and chloride, the residual

${ }^{1}$ Read before the Association of American Physicians, May 7, 1924. 
specific gravity. By plotting this fraction against the total specific gravity its percentage contribution to the whole can be made evident. If the ratio of urea plus chlorides to the other solids remains constant and the total specific gravity varies only with changes in the ratio of water to solids, then the values plotted will fall on the same percentage diagonal.

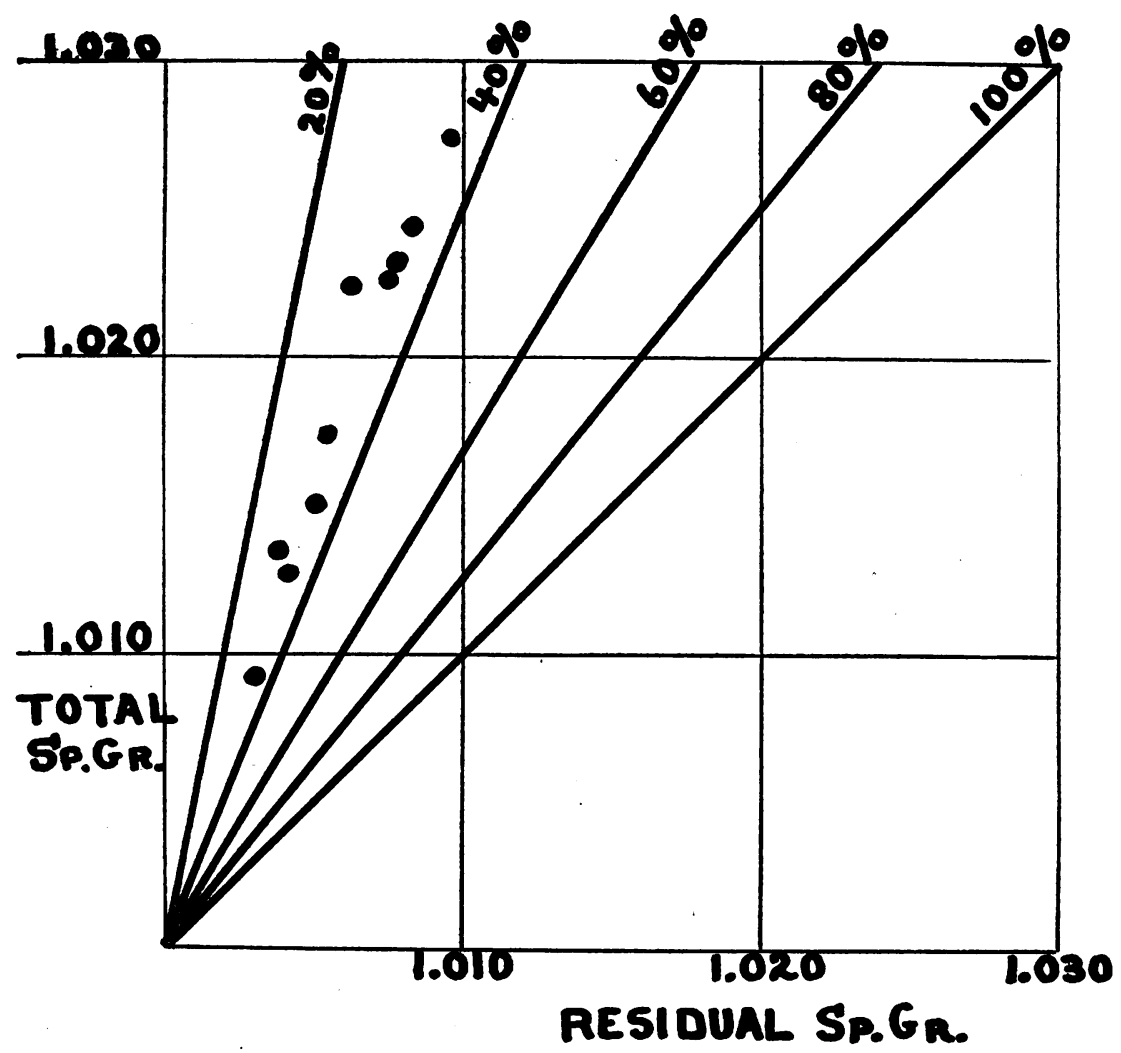

FIG. 1

Ten specimens from a normal individual, taken over a period of a year and a half are plotted in this manner in figure 1. A marked constancy of ratio is evident.

In figure 2 specimens from another normal also fall along a percentage diagonal, a little to the right of the other. In this individual the residual specific gravity forms about 42 per cent of the total. 
In figure 3 a group of thirty-six specimens from ten normals are plotted. In almost every instance the "residual" specific gravity is between 25 and 50 per cent. This constancy is striking when one considers the wide variation in total specific gravity, the number of individuals, and the uncontrolled diet.

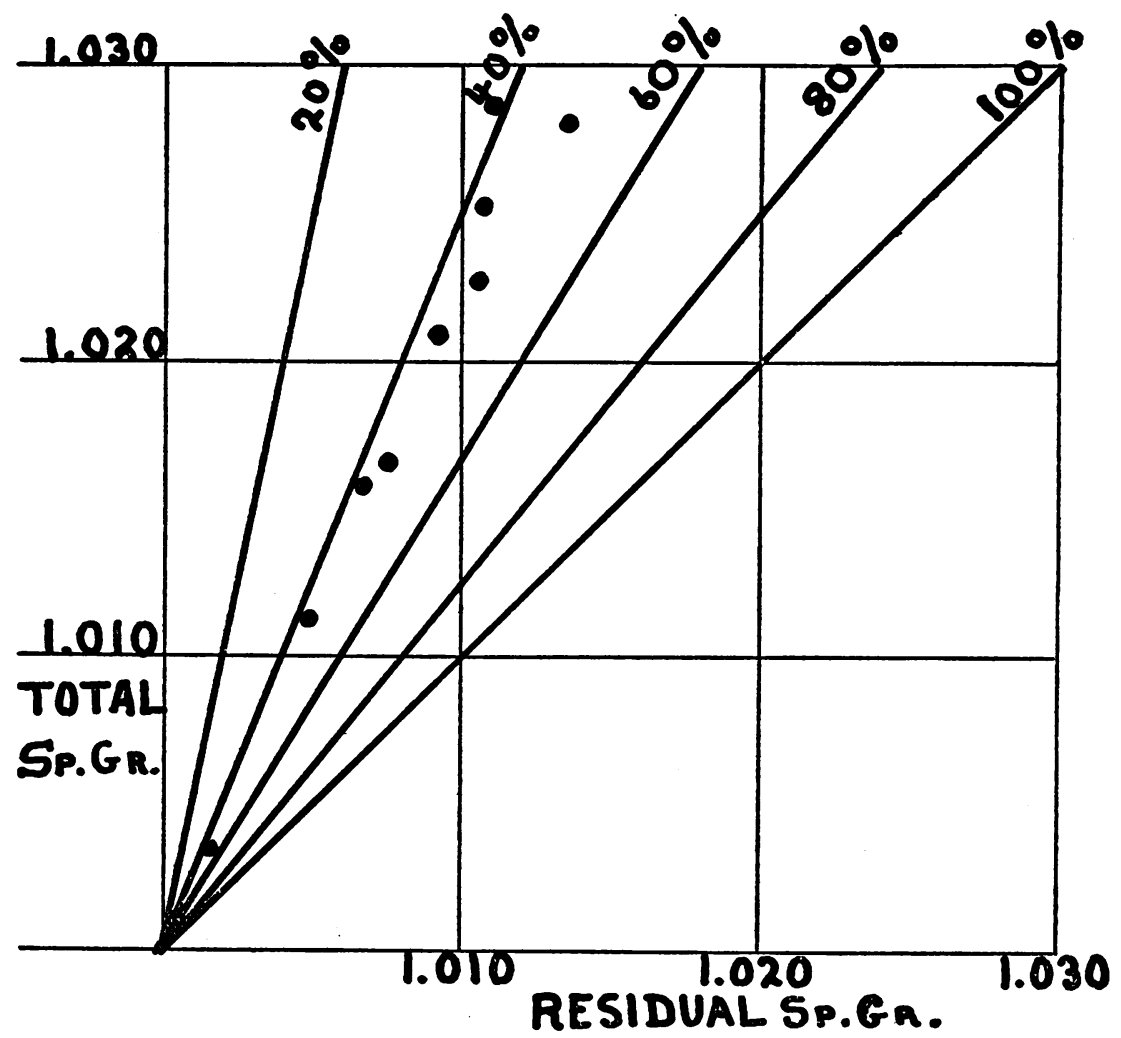

Fig. 2

This group of normals is contrasted in figure 4 with twenty specimens from twelve patients with definite chronic glomerular nephritis. In this figure the residual percentage of specific gravity is plotted against the total specific gravity. The nephritic values indicated by crosses, exhibit a ratio of residual to total specific gravity between 43 and 75 per cent. This narrow variation is the more striking in that it persisted despite efforts to vary the intake of chloride and of protein. 
In other words the solids other than the urea and chlorides make up a greater proportion of the total solids in nephritic than in normal urine. Confirmation of this is found in conductivity studies by Gram (1), working in the same laboratory, who found that in the urine of nephritics the non-chloride fraction of the electrolytes is relatively increased as compared with the chloride fraction.

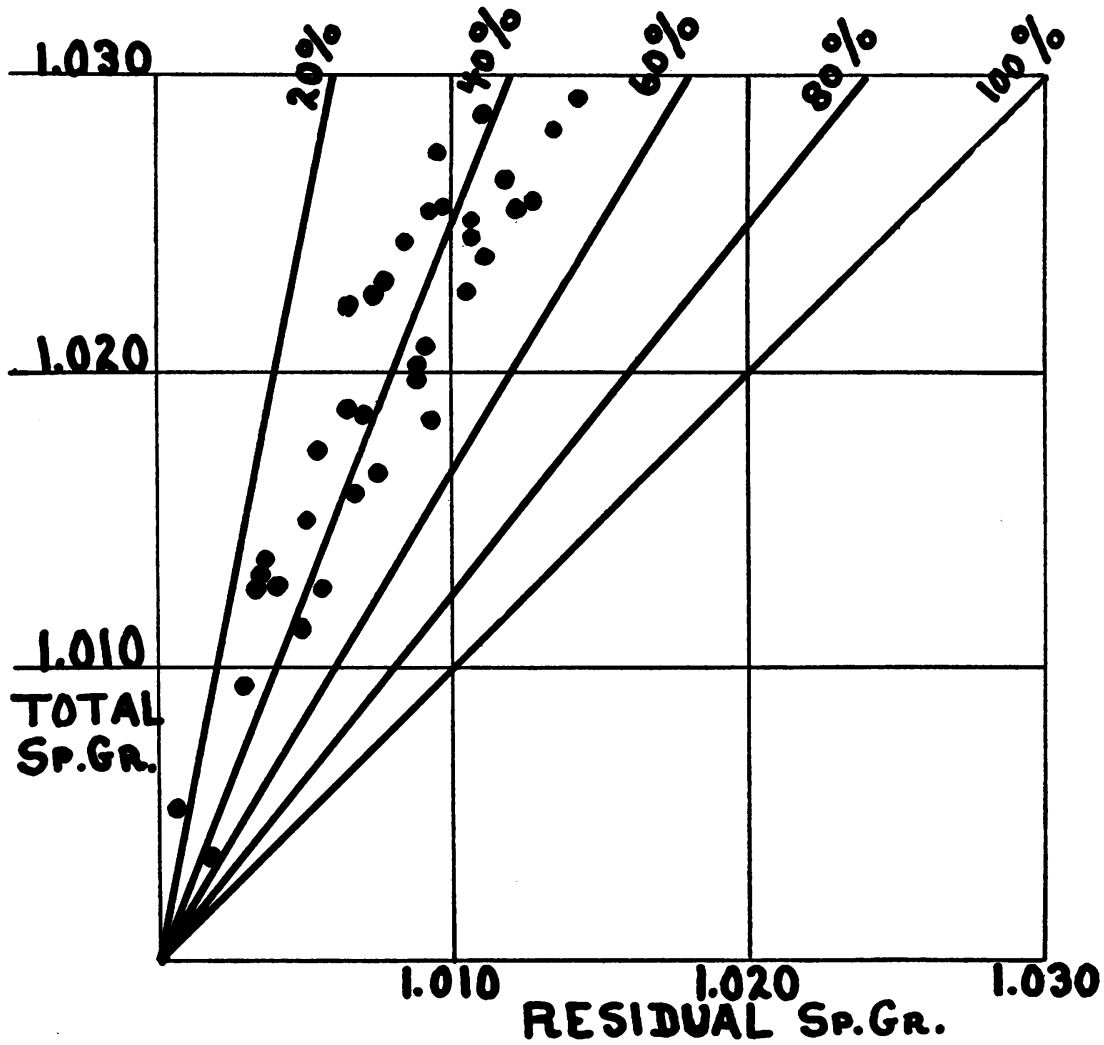

FIg. 3

Individual nephritics exhibit the same constancy of ratio as do individual normals.

The highest residual specific gravity percentages were obtained in a series of observations on a nephritic of the type with much edema. In this patient the blood pressure was normal, the 'phthalein elimination 40 per cent, the blood urea nitrogen $24 \mathrm{mg}$. and the blood cholesterol 
$730 \mathrm{mg}$. per $100 \mathrm{cc}$. The twelve values obtained from this patient are plotted in figure 5. The residual specific gravity percentage lies with great constancy between 63 and 82 . This constancy persisted in spite of changes from an almost salt free diet to a diet rich in salt,

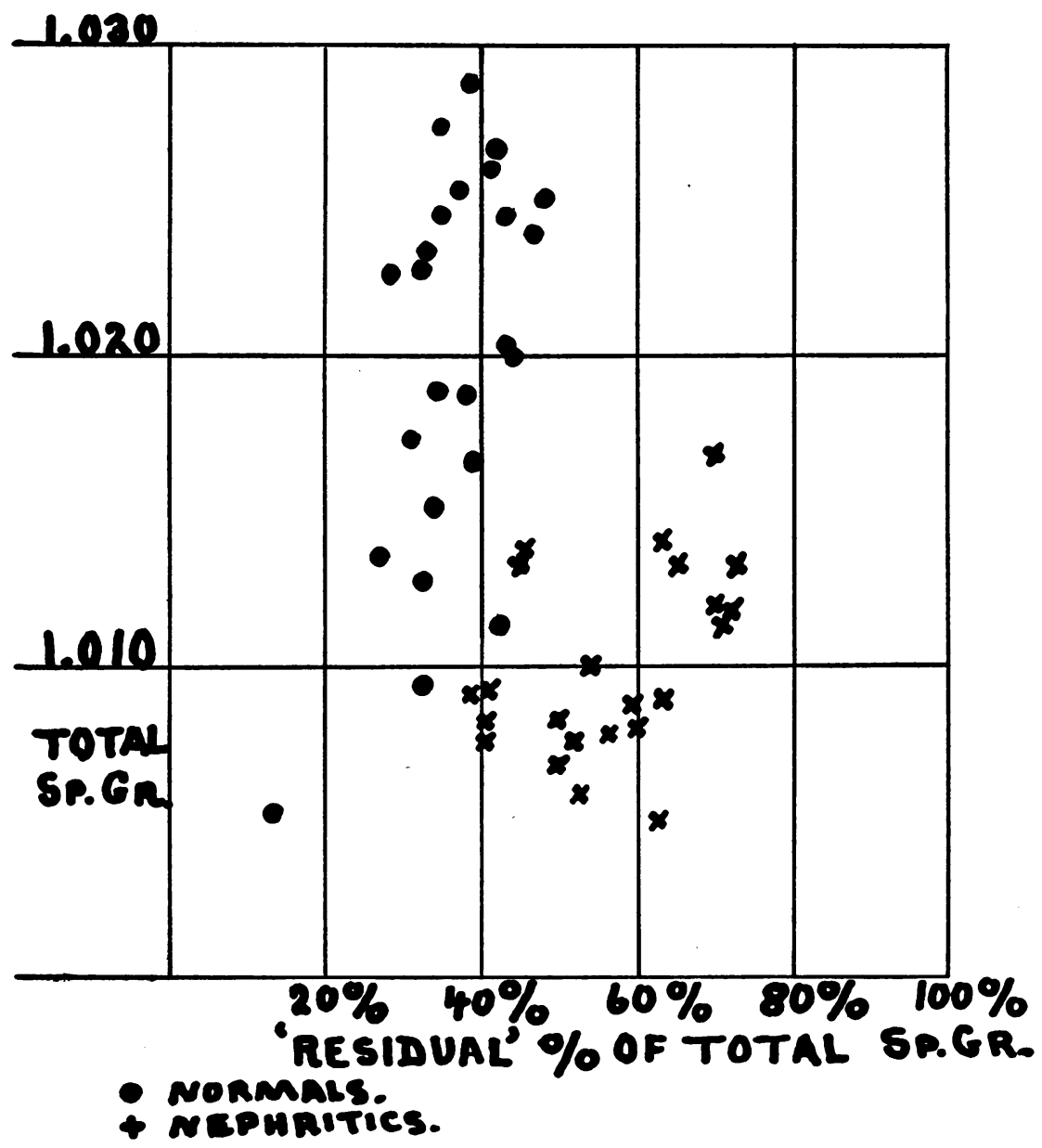

FIG. 4

and from a diet low in protein to one high in protein. The urine of this patient alone contained sufficient albumin to require consideration. This was measured by the Esbach method, converted into terms of specific gravity and subtracted from the total. A 1 per cent solution of albumin has a specific gravity of 1.0024 . 
It is interesting to note that the so-called "conductivity chloride discrepancy" which Atchley, Loeb, Benedict and Palmer (2) described in the serum of nephritics was found in this type of edematous nephritis. While it is probable that this change in conductivity should be attributed largely to the altered protein content of the

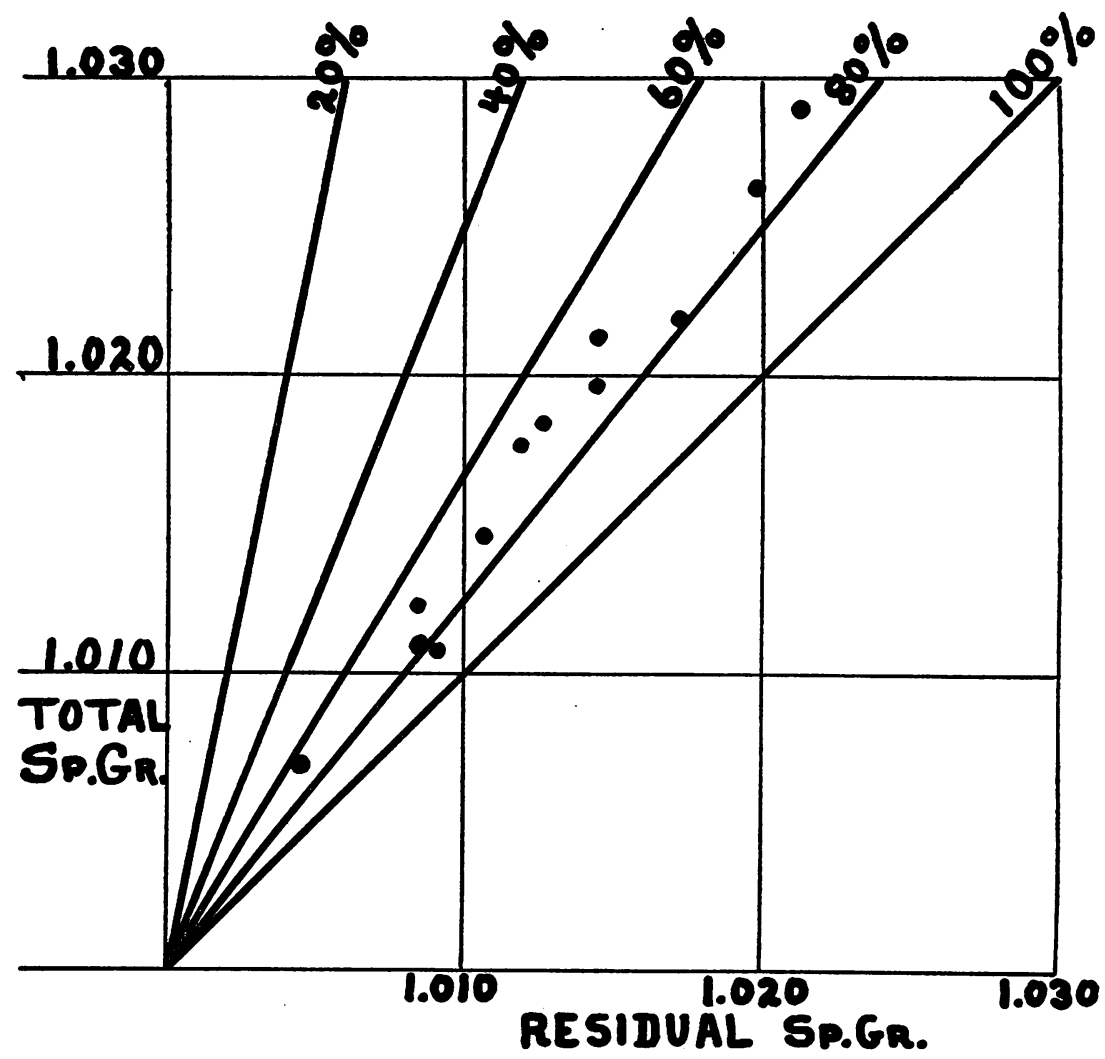

FIG. 5

serum, nevertheless it is also probable that our findings in the urine in some way reflect the altered condition of the blood.

From our observations it is clear that the nephritic fails to excrete as much urea and chlorides in a urine of a given specific gravity as would a normal. The urea plus chloride specific gravity percentage is remarkably constant in the several individuals and groups whether normal or nephritic, but the urea and chloride which form this fraction 
vary considerably in relation to one another. In the nephritics as a rule it is the chloride specific gravity which is especially reduced even when liberal salt is given in the diet. But even in the nephritic there is a considerable inverse variation of the chloride and the urea. It is our intention to study further the influence of diet, the solids forming the non-urea non-chloride fraction of the specific gravity, and the osmotic pressure and other features of the urine.

\section{SUMMARY}

When the specific gravity of the urine is analyzed into a fraction due to urea plus chlorides and the residual fraction due to the other solids, exclusive of albumin, the following relations have been observed:

1. A marked constancy of ratio between these fractions in any individual, normal or nephritic, independent of diet.

2. In normals the residual fraction forms 25 to 50 per cent of the total.

3. In 32 observations on 13 nephritics the residual fraction formed 43 to 82 per cent of the total.

4. The nephritic, independent of diet, excretes less "urea plus chlorides" in a urine of a given specific gravity than would a normal.

\section{BIBLIOGRAPHY}

1. Gram, H. C. Jour. Clinical Invest., 1924, i, 21. On the Relation Between Conductivity and Chlorides in the Urine.

2. Atchley, D. W., Loeb, R. F., Benedict, E. M., and Palmer, W. W. Arch. Int. Med., 1923, xxxi, 611. Physical and Chemical Studies of Human Serum. II. A Study of Twenty-Nine Cases of Nephritis. 